MALAYA JOURNAL OF MATEMATIK

Malaya J. Mat. 9(04)(2021), 216-221.

http://doi.org/10.26637/mjm904/005

\title{
(e)-Convergence for double sequences
}

\author{
ULAŞ YAMANCI*1 \\ ${ }^{1}$ Department of Statistics, Suleyman Demirel University, 32260, Isparta, Turkey. \\ Received 23 July 2021; Accepted 05 September 2021
}

\begin{abstract}
We define the notion of $(e)$-summability of double sequences and series of complex numbers. We also obtain a criteria for this summability method with regards to Berezin symbols of an diagonal operator, and show regularity of $(e)$ summability method for double sequences.
\end{abstract}

AMS Subject Classifications: 40H05.

Keywords: Berezin symbol, diagonal operator, double sequence, $(e)$-summability.

\section{Contents}

1 Introduction and Background 2

2 Main Results $\quad 217$

\section{Introduction and Background}

A double sequence $\left\{a_{m n}\right\}_{m, n=0}^{\infty}$ is called the convergent in Pringsheim's sense [14] provided that there exists a number $a$ such that $a_{m n}$ converges to $a$ as both $m$ and $n$ approach to infinity independently of one another

$$
\lim _{m, n \rightarrow \infty} a_{m n}=a
$$

that is if for every $\varepsilon>0$ there exists $K=K(\varepsilon) \in \mathbb{N}$ such that $\left|a_{m n}-a\right|<\varepsilon$ for every $m, n \geq K$ and also $a$ is said to the Pringsheim's limit of $a_{m n}$. It is obvious that $\left\{a_{m n}\right\}$ is convergent in Pringsheim's sense if and only if for every $\varepsilon>0$ there exists an integer $K=K(\varepsilon) \in \mathbb{N}$ such that $\left|a_{m n}-a_{i j}\right|<\varepsilon$ for $\min \{m, n, i, j\} \geq K$. A double sequence $\left\{a_{m n}\right\}$ is bounded provided that there exists a positive number $N$ such that $\left|a_{m n}\right| \leq N$ for every $m$ and $n$, i.e., $\sup _{m, n}\left|a_{m n}\right|<\infty$.

A double sequence $\left\{a_{m n}\right\}$ is said to be convergent regularly provided that it is convergent in Pringsheim's sense and the following limits hold:

$$
\begin{gathered}
\lim _{m, n \rightarrow \infty} a_{m n}=x_{m}(m=1,2, \ldots) \\
\lim _{m, n \rightarrow \infty} a_{m n}=x_{n}(n=1,2, \ldots)
\end{gathered}
$$

It is well known that a convergent double sequence in Pringsheim's sense fails in general to be bounded. The concept of regular convergence, which was introduced by Hardy in [7], lacks this advantage. Moreover, the regular convergence requires the convergence of rows and columns of a double sequence. (For more information about several type convergence for double sequences, see [18] and its references.)

*Corresponding author. Email address: ulasyamanci@sdu.edu.tr (Ulaş Yamancı) 


\section{(e)-Convergence for double sequences}

A reproducing kernel Hilbert space (shorty, RKHS) $\mathcal{H}=\mathcal{H}(\Omega)$ on some set $\Omega$ is a Hilbert space of functions on $\Omega$ such that for every $\lambda \in \Omega$ the linear functional (evaluation functional) $f \rightarrow f(\lambda)$ is bounded on $\mathcal{H}$. If $\mathcal{H}$ is RKHS on set $\Omega$, then by the classical Riesz Representation Theorem for every $\lambda \in \Omega$ there is a unique element $k_{\lambda} \in \mathcal{H}$ for which $f(\lambda)=\left\langle f, k_{\lambda}\right\rangle$ for all $f \in \mathcal{H}$. The function $k_{\lambda}$ is said to be reproducing kernel at $\lambda$. We know that (see, Aronzajn [1] and Saitoh [15]) provided that $\left(e_{j}\right)_{j \in J}$ is an orthonormal basis for the RKHS $\mathcal{H}$,

$$
k_{\lambda}(z)=\sum_{j \in J}^{\infty} \overline{e_{j}(\lambda)} e_{j}(z), \quad z \in \Omega .
$$

The function

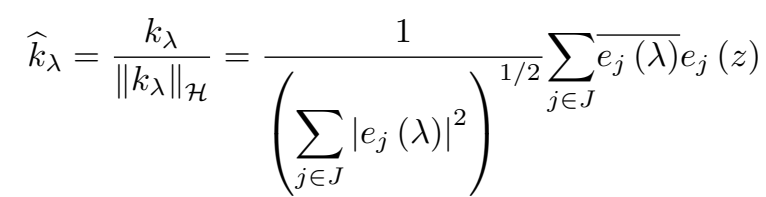

is called the normalized reproducing kernel at $\lambda$.

Berezin [2,3] introduced the concept of contravariant and covariant symbols of an operator. The contravariant symbol of a Toeplitz operator, which is the so-called Berezin symbol, was firstly used by Berger and Coburn in $[4,5]$.

Let $A$ be a bounded operator on reproducing kernel Hilbert spaces. Then the function

$$
\widetilde{A}(\lambda):=<A \widehat{k}_{\lambda}, \widehat{k}_{\lambda}>, \lambda \in \Omega,
$$

is called the Berezin symbol, which is a bounded function by the norm of the operator (see [2]). On the reproducing kernel Hilbert spaces, $\widetilde{A}_{1}(\lambda)=\widetilde{A}_{2}(\lambda)$ for all $\lambda$ implies $A_{1}=A_{2}$, that is, the Berezin symbol uniquely determines the operator. Therefore, the Berezin symbol includes many information about the operator that induces it. Prosperous applications of the Berezin symbol are up to now commonly in the study of operator theory, such as Toeplitz and Hankel operators [19]. The Berezin symbol technique is motivated by its connections with quantum physics (see, for example, [2, 3]). Readers can found more informations about Berezin symbols and its applications, for instance in $[9,13,19]$.

A RKHS $\mathcal{H}(\Omega)$ is standard provided that the underlying set $\Omega$ is a subset of a topological space and the boundary of $\Omega$ is non-empty and has the property that $\left(k_{\mathcal{H}, \lambda_{n}}\right)_{n}$ converges weakly to 0 whenever $\left(\lambda_{n}\right)_{n}$ is a sequence in $\Omega$ that converges to a point in $\partial \Omega$. It is obvious that $\lim _{n \rightarrow \infty} \widetilde{K}\left(\lambda_{n}\right)=0$ for any compact operator $K$ on the standard RKHS $\mathcal{H}$ whenever $\left(\lambda_{n}\right)_{n} \subset \Omega$ converges to a point of $\partial \Omega$. In this case, the Berezin symbol of a compact operator on a standard RKHS vanishes on the boundary (see [13]).

Karaev [11] introduced $(e)$-convergent for single sequences and series of complex numbers. Later, he [12] gave a Tauberian-type therom for $(e)$-convergent sequences. Using the Berezin symbol technique, new proofs for $(L)$-convergence and Abel convergence were given in $[8,16]$.

\section{Main Results}

In this section, we define a concept of $(e)$-convergence for double sequences and series of complex numbers. We obtain a criteria for this summability method with regards to Berezin symbols of an diagonal operator, and show regularity of $(e)$-summability method for double sequences.

Recall that a method is called the regular provided that it sums each convergent sequence to its ordinary limit. For instance, Abel, Cezaro and Borel methods are regular (see [6]).

Let $\mathcal{H}=\mathcal{H}(\Omega \times \Omega)$ be a reproducing kernel Hilbert space on some set $\Omega \times \Omega,\left\{e_{m n}\right\}_{m, n \geq 0}$ be an orthonormal basis of $\mathcal{H}$ and

$$
k_{\lambda, \mu}(z, w):=\sum_{m, n \geq 0} \overline{e_{m n}(\lambda, \mu)} e_{m n}(z, w),
$$

be a reproducing kernel of $\mathcal{H}=\mathcal{H}(\Omega \times \Omega)$. 
Definition 2.1. Let $\left\{a_{m n}\right\}_{m, n>0}$ be a double sequence of complex numbers.

(a) The sequence $\left\{a_{m n}\right\}_{m, n \geq 0}$ is (e)-convergent to L provided that

$$
\sum_{m, n=0}^{\infty} a_{m n}\left|e_{m n}(\lambda, \mu)\right|^{2}
$$

converges for all $(\lambda, \mu) \in \Omega \times \Omega$ and

$$
\lim _{(\lambda, \mu) \rightarrow(\zeta, \xi)} \frac{1}{\sum_{m, n=0}^{\infty}\left|e_{m n}(\lambda, \mu)\right|^{2}} \sum_{m, n=0}^{\infty} a_{m n}\left|e_{m n}(\lambda, \mu)\right|^{2}=L,
$$

for every $(\zeta, \xi) \in \partial \Omega \times \partial \Omega$.

(b) The series $\sum_{m, n=0}^{\infty} a_{m n}$ is (e)-summable to L provided that

$$
\sum_{m, n=0}^{\infty} a_{m n}\left|e_{m n}(\lambda, \mu)\right|^{2}
$$

converges for each $(\lambda, \mu) \in \Omega \times \Omega$ and

$$
\lim _{(\lambda, \mu) \rightarrow(\zeta, \xi)} \sum_{m, n=0}^{\infty} a_{m n}\left|e_{m n}(\lambda, \mu)\right|^{2}=L
$$

for each $(\zeta, \xi) \in \partial \Omega \times \partial \Omega$.

It was shown that Abel and Borel summability for double sequences coincide with concept of $(e)$-summability for Hardy space and Fock space, respectively (see [10, 17]).

Let $\left\{a_{m n}\right\}_{m, n \geq 0}$ be a double sequence of complex numbers. Diagonal operator $D_{\left\{a_{m n}\right\}}$ on $\mathcal{H}$ is defined by

$$
D_{\left\{a_{m n}\right\}} e_{m n}(\lambda, z)=a_{m n} e_{m n}(\lambda, z), m, n=0,1,2, \ldots
$$

with respect to the orthonormal basis $e=\left\{e_{m n}(\lambda, z)\right\}_{m, n \geq 0}$ of $\mathcal{H}$.

The following result is main theorem of this section.

Theorem 2.2. Let $\left\{a_{m n}\right\}_{m, n \geq 0}$ be a bounded double sequence of complex numbers.

(a) The sequence $\left\{a_{m n}\right\}_{m, n \geq 0}$ is $(e)$-convergent to $L$ if and only if

$$
\lim _{(\lambda, \mu) \rightarrow(\zeta, \xi)} \widetilde{D}_{\left\{a_{m n}\right\}}(\lambda, \mu)=L
$$

for every $(\zeta, \xi) \in \partial \Omega \times \partial \Omega$.

(b) The series $\sum_{m, n=0}^{\infty} a_{m n}$ is (e)-summable to $L$ if and only if

$$
\lim _{(\lambda, \mu) \rightarrow(\zeta, \xi)}\left(\sum_{m, n=0}^{\infty}\left|e_{m n}(\lambda, \mu)\right|^{2}\right) \widetilde{D}_{\left\{a_{m n}\right\}}(\lambda, \mu)=L
$$

for every $(\zeta, \xi) \in \partial \Omega \times \partial \Omega$.

(c) (e)-summability method for double sequences is regular provided that $\mathcal{H}$ is a standard functional Hilbert space. 
(e)-Convergence for double sequences

Proof. As $\left\{a_{m n}\right\}_{m, n \geq 0}$ is a bounded double sequence, $D_{\left\{a_{m n}\right\}}$ is a bounded operator on $\mathcal{H}$. Calculating the Berezin symbol of diagonal operator, we have

$$
\begin{aligned}
\widetilde{D}_{\left\{a_{m n}\right\}}(\lambda, \mu) & =\left\langle D_{\left\{a_{m n}\right\}} \widehat{k}_{\lambda, \mu}, \widehat{k}_{\lambda, \mu}\right\rangle \\
& =\frac{1}{\left\|k_{\lambda, \mu}\right\|^{2}}\left\langle D_{\left\{a_{m n}\right\}} \sum_{m, n=0}^{\infty} \overline{e_{m n}(\lambda, \mu)} e_{n}(z, w), k_{\lambda, \mu}\right\rangle \\
& =\frac{1}{\sum_{m, n=0}^{\infty}\left|e_{m n}(\lambda, \mu)\right|^{2}}\left\langle\sum_{m, n=0}^{\infty} \overline{e_{m n}(\lambda, \mu)} a_{m n} e_{m n}(z, w), k_{\lambda, \mu}\right\rangle \\
& =\frac{1}{\sum_{m, n=0}^{\infty}\left|e_{m n}(\lambda, \mu)\right|^{2}} \sum_{m, n=0}^{\infty} a_{m, n}\left|e_{m n}(\lambda, \mu)\right|^{2}
\end{aligned}
$$

for all $(\lambda, \mu) \in \Omega \times \Omega$. Therefore

$$
\widetilde{D}_{\left\{a_{m n}\right\}}(\lambda, \mu)=\frac{1}{\sum_{m, n=0}^{\infty}\left|e_{m n}(\lambda, \mu)\right|^{2}} \sum_{m, n=0}^{\infty} a_{m n}\left|e_{m n}(\lambda, \mu)\right|^{2},(\lambda, \mu) \in \Omega \times \Omega .
$$

As $\sup _{(\lambda, \mu) \in \Omega \times \Omega}\left|\widetilde{D}_{\left\{a_{m n}\right\}}(\lambda, \mu)\right| \leq\left\|\widetilde{D}_{\left\{a_{m n}\right\}}\right\|=\sup _{m, n \geq 0}\left|a_{m n}\right|<\infty$, formula (1) immediately implies the claims (a) and (b) of the theorem.

Let us show the claim (c). Let $\left\{a_{m n}\right\}_{m, n=0}^{\infty}$ converges to $L$. Then $D_{\left\{a_{m n}-L\right\}}$ is a compact operator, and hence $\widetilde{D}_{\left\{a_{m n}-L\right\}}$ vanishes on the boundary of $\Omega \times \Omega$ (since $\mathcal{H}$ is a standard reproducing kernel Hilbert space), that is, $\widetilde{D}_{\left\{a_{m n}-L\right\}}(\lambda, \mu) \rightarrow 0$ as $(\lambda, \mu) \rightarrow(\zeta, \xi) \in \partial \Omega \times \partial \Omega$. Taking into consideration this and formula (1), we get

$$
\begin{aligned}
\lim _{(\lambda, \mu) \rightarrow(\zeta, \xi)} \widetilde{D}_{\left\{a_{m n}\right\}}(\lambda, \mu) & =\lim _{(\lambda, \mu) \rightarrow(\zeta, \xi)} \frac{1}{\sum_{m, n=0}^{\infty}\left|e_{m n}(\lambda, \mu)\right|^{2}} \sum_{m, n=0}^{\infty} a_{m n}\left|e_{m n}(\lambda, \mu)\right|^{2} \\
& =\lim _{(\lambda, \mu) \rightarrow(\zeta, \xi)} \frac{1}{\sum_{m, n=0}^{\infty}\left|e_{m n}(\lambda, \mu)\right|^{2}} \sum_{m, n=0}^{\infty}\left(a_{m n}-L+L\right)\left|e_{m n}(\lambda, \mu)\right|^{2} \\
& =\lim _{(\lambda, \mu) \rightarrow(\zeta, \xi)} \frac{1}{\sum_{m, n=0}^{\infty}\left|e_{m n}(\lambda, \mu)\right|^{2}} \sum_{m, n=0}^{\infty}\left(a_{m n}-L\right)\left|e_{m n}(\lambda, \mu)\right|^{2}+L \\
& =\lim _{(\lambda, \mu) \rightarrow(\zeta, \xi)} \widetilde{D}_{\left\{a_{m n}-L\right\}}+L,
\end{aligned}
$$

which gives that $(e)-\lim _{m, n} a_{m n}=L$. So, the proof is completed.

We can obtain the following result from Theorem 1 by putting $\mathcal{H}=\mathcal{D}\left(\mathbb{D}^{2}\right)$ and $\mathcal{H}=\mathcal{F}\left(\mathbb{C}^{2}\right)$.

Corollary 2.3. Let $\left\{a_{m n}\right\}_{m, n \geq 0}$ be a bounded double sequence of complex numbers.

(a) If $D_{\left\{a_{m n}\right\}}$ is a diagonal operator on the Dirichlet space $\mathcal{D}\left(\mathbb{D}^{2}\right)$ with diagonal elements $a_{m n}, m, n \geq 0$, with respect to the orthonormal basis of $\mathcal{D}$, then the double sequence $\left\{a_{m n}\right\}_{m, n \geq 0}$ is $(L)$-convergent (logarithmic convergent) to $L$ if and only if

$$
\lim _{\lambda, \mu \rightarrow 1^{-}} \widetilde{D}_{\left\{a_{m n}\right\}}(\sqrt{x}, \sqrt{y})=L,
$$

where $x=|\lambda|^{2}$ and $y=|\mu|^{2}$.

(b) If $D_{\left\{a_{m n}\right\}}$ is a diagonal operator on the Fock space $\mathcal{F}\left(\mathbb{C}^{2}\right)$ with diagonal elements $a_{m n}, m, n \geq 0$, with 
respect to the orthonormal basis of $\mathcal{D}$, then the double sequence $\left\{a_{m n}\right\}_{m, n \geq 0}$ is Borel convergent to $L$ if and only if

$$
\lim _{\lambda, \mu \rightarrow \infty} \widetilde{D}_{\left\{a_{m n}\right\}}(\sqrt{2 x}, \sqrt{2 y})=L
$$

where $x=\frac{|\lambda|^{2}}{2}$ and $y=\frac{|\mu|^{2}}{2}$.

\section{References}

[1] N. AronZajn, Theory of reproducing kernels, Trans. Amer. Math. Soc., 68(1950), 337-404.

[2] F.A. BEREZIN, Covariant and contravariant symbols for operators, Math. USSR-Izv., 6(1972), 1117-1151.

[3] F.A. BEREZIN, Quantization, Math. USSR-Izv., 8(1974), 1109-1163.

[4] C.A. Berger And L.A. Coburn, Toeplitz operators and quantum mechanics, J. Funct. Anal., 68 (1986), 273-299.

[5] C.A. Berger and L.A. Coburn, Toeplitz operators on the Segal-Bergman space, Trans. Amer. Math. Soc., 301 (1987), 813-829.

[6] G.H. Hardy, Divergent Series, Oxford, 1956.

[7] G.H. Hardy, On the convergence of certain multiplie series, Proc. Cambridge Philos. Soc., 19 (1916-1919), 86-95.

[8] M.T. Karaev, Functional analysis proofs of Abel's theorems, Proc. Amer. Math. Soc., 132 (2004), 23272329.

[9] M.T. Karaev, On some problems related to Berezin symbols, C. R. Acad. Sci. Paris, 340(2005), 715-718.

[10] M.T. Karaev and M. Zelster, On Abel convergence of double sequences, Numer. Funct. Anal. Optim., 31(10)(2010), 1185-1189.

[11] M.T. Karaev, (e)-convergence and related problem, C. R. Math. Acad. Sci. Paris, 348(2010), 1059-1062.

[12] M.T. Karaev, Tauberian-type theorem for (e)-convergent sequences, C. R. Math. Acad. Sci. Paris, 351(2013), 177-179.

[13] E. Nordgren And P. Rosenthal, Boundary values of Berezin symbols, Operator Theory: Advances and Applications, 73(1994), 362-368.

[14] A. Pringsheim, Elementare theorie der unendliche doppelreihen, Sitsungs Berichte der Math. Akad. der Wissenschafften zu Münch. Ber., 7(1898), 101-153.

[15] S. SAIтоH, Theory of reproducing kernels and its applications, Pitman Research Notes in Mathematics Series, v.189, 1988.

[16] U. Yamanci, On the summability methods of logarithmic type and the Berezin symbol, Turk. J. Math., 42(2018), 2417-2422.

[17] U. Yamanci, On Borel convergence of double sequences, Commun. Fac. Sci. Univ. Ank. Ser. Al Math. Stat., 68(2)(2019), 1289-1293.

[18] M. Zelster, Investigation methods for summability of double sequences, Ph.D thesis, Tallin, 2001. 


\section{(e)-Convergence for double sequences}

[19] K. ZHu, Operator Theory in Function Spaces, New York: Marcel Dekker, 1990.

This is an open access article distributed under the Creative Commons Attribution (c) (i) License, which permits unrestricted use, distribution, and reproduction in any medium, provided the original work is properly cited. 